\title{
Holistic System-Analytics as an Alternative to Isolated Sensor Technology: A Condition Monitoring Use Case
}

\author{
Dominik Martin \\ Karlsruhe Institute of Technology (KIT) \\ $\underline{\text { martin@kit.edu }}$
}

\begin{abstract}
Sensor technology has become increasingly important (e.g., Industry 4.0, IoT). Large numbers of machines and products are equipped with sensors to constantly monitor their condition. Usually, the condition of an entire system is inferred through sensors in parts of the system by means of a multiplicity of methods and techniques. This so-called condition monitoring can thus reduce the downtime costs of a machine through improved maintenance scheduling. However, for small components as well as relatively inexpensive or immutable parts of a machine, sometimes it is not possible or uneconomical to embed sensors. We propose a system-oriented concept of how to monitor individual components of a complex technical system without including additional sensor technology. By using already existing sensors from the environment combined with machine learning techniques, we are able to infer the condition of a system component, without actually observing it. In consequence condition monitoring or additional services based on the component's behavior can be developed without overcoming the challenges of sensor implementation.
\end{abstract}

\section{Introduction and Motivation}

With the rapid development of advanced sensor technology and the decline in hardware costs an increasing number of machines and products has been equipped with sensors to acquire data on assets behavior $[1,2,3]$. Condition monitoring uses this data in order to facilitate the detection of machine malfunctions in an early state, and, thus, minimizing consequential damage, enhancing maintenance work scheduling as well as reducing downtime costs [4].

Industry as well as academia face the challenge of developing condition monitoring solutions for machines, products, systems and components. Most research focuses on the development of smart materials equipped with sensors, or equipping machines or

\author{
Niklas Kühl \\ Karlsruhe Institute of Technology (KIT) \\ $\underline{\text { kuehl@kit.edu }}$
}

components with a large number of sensors designed for this particular purpose $[5,6,7]$.

However, in many cases it is either not technically feasible or uneconomic to monitor the components or parts of interest with dedicated sensors [8, 9, 10]. For instance, it is not possible to incorporate sensor technology into a part of interest-such as i.e., a seal-due to, for instance, technical reasons or unreasonable expenses.

Especially for very complex assets (i.e., aircraft engines, production machines, wind turbines), the reliability of each individual component is of enormous importance. Such complex assets consist of a number of components that often again are composed of subcomponents produced by various manufacturers. The manufacturing at the entire system level thus requires only little knowledge about the behavior of all parts down to the smallest components. Therefore, the OEMs do not necessarily have the technical know-how to develop high-quality condition monitoring solutions for each individual component.

Nevertheless, it may be interesting to monitor even small and seemingly negligible parts in order to gain insights about the part's behavior within a complex environment, to differentiate from competitors or to be able to offer additional services. Such services could be, for instance, maintenance services, wear prediction or analysis tools for a specific component or part. Even condition monitoring as a service could be a conceivable business model. Furthermore, condition monitoring allows to prevent consequential damage to the overall system due to a component malfunction. However, especially for suppliers of very cheap or small components, it is often not possible to offer their own condition monitoring solutions for their products, since an observation of these components in the context of the overall system is too costly. Thus, for instance, embedding special sensors into a part or even customize the surrounding system of a component that cost a few cents is generally not profitable.

In this work, we propose a concept to observe the 
state of individual parts by a system-wide consideration without actually being able to observe them directly.

By applying machine learning techniques, we show how conclusions about individual components can be drawn by considering the entire system. Thus, our approach allows to make statements about the behavior or condition of subordinate parts without additional hardware being necessary.

We show that, despite complex interrelations within a system, as described by General Systems Theory [11, 12] and the idea of holism [13, 14], condition monitoring of components of a system can be accomplished by means of machine learning.

\section{Foundations and Related Work}

We consider machines, products and assets as systems and therefore use systems theory-an interdisciplinary approach for describing and explaining aspects, properties and principles of systems-to describe interrelations and the behavior of systems and its components. Subsequently, we introduce the concept of condition monitoring and discuss related literature regarding existing condition monitoring approaches.

\subsection{Systems Theory}

The aim of classical physics was to reduce natural phenomena to an interaction of elementary units-such as atoms-whose properties are independent of the environment, and thus of adjacent units. Similarly, in biology, the assumption was that phenomena of life can be resolved into separate parts that can be considered in isolation. Accordingly, an organism can be thought of as the interplay of various elements-such as cells- that function independently [11].

This traditional scientific theory for exploring single components without regard to the surrounding units is known as reductionism. Reductionism is a bottom-up approach and tries to deduce the understanding of the whole from the understanding of individual parts. However, a whole aggregated of parts is not immediately apparent from the individual parts [15].

This problem arises from complex interrelationships between the individual parts, from which a whole emerges. Only the whole gives the parts and their interactions a meaning. For instance, only a living organism gives meaning to the heart or lungs as well as a family to the roles of husband, wife, son or daughter [16].

According to this assumption, and contrary to the idea of reductionism, modern biology has developed the notion that not only the consideration of isolated parts, but also of relations between parts and the resulting dynamic interactions is essential. This results in differences between individual parts considered separately and parts within a whole organism [11, 12].

The biologist Ludwig von Bertalanffy proposed systems theory in the 1940's, in which he transferred and generalized this idea to other scientific disciplines [17]. He defines systems as interaction contexts that differ from their environment, which in turn consists of other interaction contexts. Through their interaction with the environment, they can evolve new properties. System theory does not reduce the whole (i.e., the human body) to the behavior or properties of its parts (i.e., organs), but rather describes the relationships and arrangement of the parts which build the whole [17].

Accordingly, holism is the idea that systems (biological, physical, social, economic, etc.) and their properties should be viewed as wholes, not just as a collection of parts [13, 14].

Based on this, Ackoff [18] defines a system as a set of two or more elements that satisfies following conditions: The behavior of each element has an effect on the behavior of the whole, where both the behavior of the elements as well as their effects on the whole are interdependent. Additionally, all subgroups of elements have an effect on the behavior of the whole but none has an independent effect on it.

\subsection{Condition Monitoring}

Observing the behavior of systems and their elements poses a complex challenge in various disciplines, such as biology, physics, medicine, but also in computer science or mechanical engineering. Especially in industry, the continuous observation of machine or product conditions is an important factor for smooth operations.

The concept of condition monitoring describes the regular acquisition and analysis of physical parameters for monitoring the condition of machines. The physical machine parameters are collected by means of sensors and include vibration [19, 20], temperature [21, 22], acoustic emission [23, 24], electricity [25], and many more.

By identifying significant changes in the sensor readings, the occurrence of machine failures is determined by a variety of methods [26]. This makes condition monitoring an important basis for predictive maintenance. By being aware of the machine conditions at any time, maintenance work can be better planned and accordingly machine downtimes reduced [26]. In addition, further actions can be made to minimize consequential damage as well as the effects of a failure on surrounding components. Condition monitoring 
allows the earlier detection of machine failures, before their effects can be directly perceived by humans. Thus, condition monitoring is an effective alternative to periodic machine inspections [27].

Especially with rotating machines, such as motors, pumps, and compressors, condition monitoring methods can be used effectively [20]. However, interesting research approaches are also available for translatory or even static applications.

For instance, Nandi et. al. [26] describe different components which indicate failures in electric motors. In addition to the typical failure types occurring in different components, common recognition and analysis techniques are summarized. However, the focus relies on the analysis of conditions of the entire electric motor system. A targeted analysis of components such as the bearing or even components of it, without dedicated sensors, is not considered.

Also, Carden and Fanning [20] summarize different methods and techniques which appear in literature, which are capable of detecting failures based on the analysis of vibrations. They argue that different methods require a varying number of sensors and the results improve as the number of sensors increases.

This work does neither want to treat the integration of new sensors into a system, nor to consider the behavior of the whole system. Our work focuses on monitoring a single component of a system that does not have integrated sensing capabilities by using already available sensors from the component's environment. Thus, we aim to show the feasibility of inferring the condition of such a system component even without dedicated embedded sensors.

\section{Research Design}

Our goal is to present a concept to gain insights about the behavior of a part of a system based on data gathered from sensors in the part's environment. Thus, our first research question is:

RQ1: How can we design a concept that allows us to derive the behavior of a part of a system based on system-wide considerations, without being able to observe this directly?

We follow the research paradigm of Design Science Research, which is widely accepted in the field of information systems [28, 29]. The central idea of the Design Science Research approach is to gain an understanding of the problem domain and scientifically founded knowledge while at the same time guaranteeing practical relevance [30]. This is achieved by developing an artifact. Our artifact is a construct [31], which is evaluated by a technical experiment [28]. Thus, our knowledge contribution is thereby an exaptation, since we extend already known solutions (application of General Systems Theory) to new problems (system-based condition monitoring for components) [29].

By developing an artifact in a first design cycle, we aim to show the feasibility of inferring the behavior of a single component within a system without actually observing it, using already available sensors.

Based on this concept, we aim to develop a condition monitoring system artifact for hydraulic cylinders, which is capable of detecting seal failures on the basis of sensors within the cylinder, but, however, without being able to observe the seal directly. Thus, we want to evaluate the feasibility of our proposed concept by means of a concrete technical experiment. Therefore, the second research question is as follows:

RQ2: By applying a systemic view on the hydraulic cylinder system, can we infer the state of individual components without directly monitoring them itself?

After demonstrating the feasibility, we also provide a prototype that demonstrates a possible use case.

\section{Concept of Holistic System-Analytics}

As described by Ackoff [18], systems consist of several elements that are exposed to each other's interactions and thus influence the behavior of the entire system. According to the idea of holism, the total system is more than the sum of its parts. This contradicts the approach of reductionism, which intends to analyze all individual components of a system in order to comprehensively describe the behavior of the entire system. Based on these assumptions, the behavior of a system from a holistic viewpoint can not be fully explained by the behavior of the individual elements. Thus, no direct inferences from the overall system behavior to its elements are possible, since the behavior of the entire system is influenced by complex interdependent relationships of the system elements.

However, even if there is no direct relationship between the behavior of the entire system and individual elements of it, each of the effects of an element influences the behavior of the entire system. Thus, by observing these more complex relationships on a system level, patterns can be recognized which are caused by individual elements.

Typically, monitoring of technical systems is achieved by incorporating sensors and observation capabilities into its components. Individual components in complex systems are analyzed separately. Based on this information, conclusions about the behavior of the entire system are derived [26]. 
Figure 1 visualizes this common approach of a system-level analysis. It shows an open system, which consists of a set of elements having interdependent effects on the entire system. The system itself also interacts with the environment. Individual elements of the system can be observed by i.e., sensors (elements A and $\mathrm{C}$ ), while other elements are unobservable (element B). Using the observable behavior of elements and the observable interaction of the entire system with the environment, conclusions can be drawn about the behavior of the entire system.

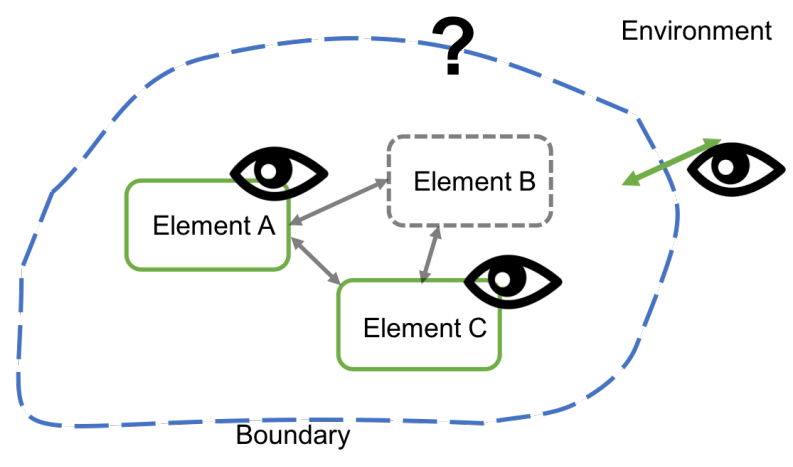

Figure 1. System-level analysis

Our proposed concept describes a contradictory approach. This concept describes a method to analyze elements of a complex technical system in which an element of interest on the one hand is not directly observable and on the other hand has no trivial connections between the behavior of itself and the behavior of the entire system. Based on data representing information about the behavior of the entire system, machine learning is used to identify the effects of individual components on the entire system. This approach allows to observe the behavior and thus the state of system components, which are not observable directly.

This approach is visualized in figure 2. Therefore, the behavior of an element within the entire system is of interest. However, this behavior can not be observed directly. Thus, by observing the interaction of the system with the environment, the impact of the element's behavior (element B) on these interactions is inferred.

This systemic top-down approach, in which the behavior of the entire system serves as basis for the analysis of system elements, requires a technique to identify patterns in the interactions of the system with the environment and, to isolate the corresponding causers of these patterns.

We use machine learning techniques for tackling

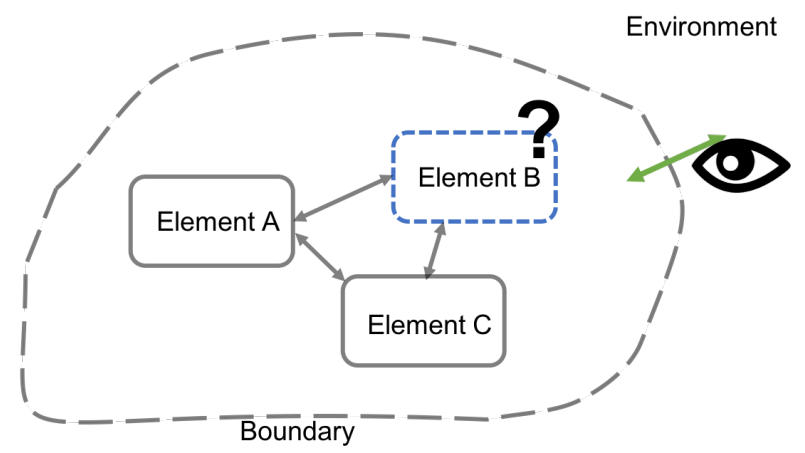

Figure 2. Component-level analysis

this and demonstrate the feasibility of our approach by means of an industrial use case. Thus, our proposed concept of compensating missing sensors in components by applying machine learning techniques to infer component behavior represents an Design Science Research artifact.

\section{Evaluation and Discussion}

This section evaluates the described artifact (presented concept). Therefore, we use an industrial use case to assess the feasibility of our approach. We define a hydraulic cylinder as a system in which a seal (component) is to be observed without equipping it with sensors.

\subsection{Technical Experiment}

A hydraulic system is used to transfer power from a hydraulic pump to a piston. By introducing a pressurized hydraulic fluid into a cylinder, the piston within the cylinder is moved. The medium necessary for this is a hydraulic fluid, such as oil, which is usually heavily pressurized. Depending on the application (i.e., an excavator) enormous forces act on the piston that are transmitted from the hydraulic system. The seals which seal the piston rod to the cylinder wall are thus essential for the function of such a hydraulic system.

Seals, even if they seem unimportant and inconspicuous, are an essential component of various applications. The catastrophic incident of the NASA space shuttle Challenger in 1986 caused by a damaged seal, reveals that seal failures can lead to dramatic effects [27].

As described in section 3, we develop a machine learning model, which is able to infer the behavior, or the state of a seal within a hydraulic cylinder based on sensor time series obtained from the surrounding of the seal itself. 
The intention of this model is the real-time detection of failure scenarios. Seals are sensitive components of hydraulic applications. Thus, a malfunction of sealing elements results in inadequate sealing efficiency and thus - in extreme cases - in a failure of the entire application. Due to the high sensitivity, a correct installation of an undamaged seal must be ensured. The model has to be capable to detect i.e., a possible assembly failure or a damaged seal.

Thus, we define three failure classes: no failure, assembly failure and damage. Data gained from different tests conducted on hydraulic cylinder test rigs serves as basis for the machine learning model, which is able to recognize patterns in the data and derive a class assignment accordingly. In order to obtain a wide range of different failure scenarios, various assembly failures and damages are simulated in several test series.

In order to train the classification model 400 hours of data with a frequency of $20 \mathrm{~Hz}$ captured by 13 sensors is available.

These sensors capture different pressures, temperatures, and rod velocity of the hydraulic cylinder. The data is cleaned and features are extracted. The failure classes are used as labels.

We use a Random Forest Classifier, as it achieves good performance in pre-tests with low training time. For model training and validation by conducting a grid search, we use 10-fold nested cross validation to avoid overfitting [32].

By using the F1-score [33] as a performance metric, the classification model achieves an average score of 0.971. In addition, we use neural networks, decision trees, and support vector machines as classification algorithms, all of which, however, yield lower performance (below 0.94).

\subsection{Prototype}

As described in section 5.1 the condition of seals can be classified by the application of machine learning algorithms. This proves the general feasibility of inferring the condition of seals without directly implemented sensors. This opens up the possibility of making this knowledge accessible and usable for third parties (i.e., customers). Therefore, we deploy the previously developed model as a web service, thus, it can be accessed via internet.

The architecture of this prototype is shown in figure 3. The raw data collected at the hydraulic cylinder is provided unprocessed to an IoT gateway. This gateway allows secure and scalable communication between IoT devices and the cloud, and makes the data available to downstream processes, like stream

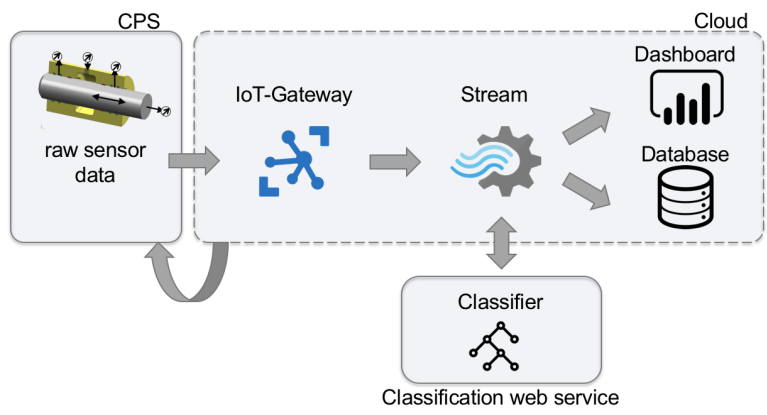

Figure 3. Architecture of condition monitoring prototype

processing. Streaming allows data to be processed in real-time and delivered to the deployed model realized by a RESTful web service. The web service handles the data cleaning and feature extraction and uses the implemented classifier to determine the corresponding failure class of the seal. This information is passed back to the stream module as a response. Subsequently, the raw data, extended to the determined failure information is passed to the dashboard module. This allows the visualization of real-time data in a browser. In addition, the raw data, extended by the classification results, is stored in a database. Optionally, the determined classification results can be transmitted back to the hydraulic cylinder's control unit in order to initiate appropriate actions there. For practical applications, changing load conditions or switching off the affected cylinder would be conceivable.

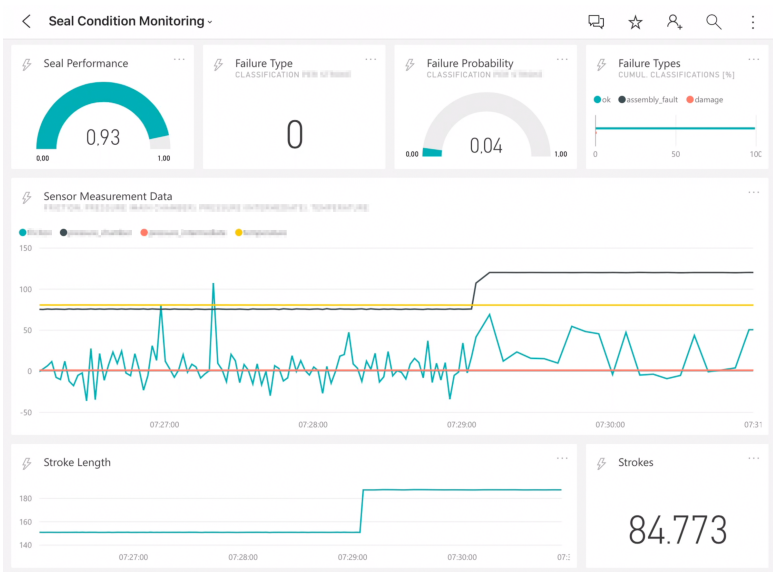

Figure 4. Screenshot of condition monitoring dashboard prototype

Figure 4 shows a screenshot of the dashboard showing the condition of the sealing elements in real-time. Thus, visual monitoring of the seal condition is possible with a simple graphical interface. The first 
tile at the top left shows the current seal condition on a virtual scale from 0 to 1 . This is an indicator calculated from the prediction probability and the moving average of historical inferences. The second tile shows the current classified seal condition and the third tile the corresponding probability of a seal failure in the present classification result. The last tile in the first row shows the relative frequency of classified failure classes since system ramp-up. The lower part of the dashboard shows current sensor raw data in real time.

\subsection{Discussion}

This work shows how machine learning can be used to obtain insights about the behavior of a system component by data obtained from system-level sensors.

Thus, there are some practical implications. Component manufacturers which previously provided only physical products thus have the opportunity to monetize their domain know-how and offer additional services supporting their core products [34]. However, collaboration with data owners and manufacturers of the immediate environment of the part or component, including the sensors, is required. Embedding component services to the entire system level, however, also creates additional value concerning the entire system. Furthermore, failure localization within a system is a complex problem, which could be solved hereby. In general, failures are only analyzed at the system level. Thus, an investigation on failure causes needs to be conducted additionally. Knowing the exact location of a failure within a complex system can therefore lead to less time-consuming troubleshooting [35].

\section{Conclusion and Outlook}

Due to embedded sensors, machines and products produce large amounts of data which is often only used for a specific purpose. The entire potential of this already existing data is therefore far from being exploited. Furthermore, some manufacturers face the challenge that some components of complex technical systems can not be monitored because no sensors can be implemented for technical or economic reasons.

Therefore we propose a concept how this problem can be resolved. By means of a system-wide analysis of existing sensor data from the immediate environment of the part of interest through machine learning, it is possible to extract patterns that can be used to deduce the behavior of parts of a system.

Based on an industrial use case, we show that the condition of a seal within a hydraulic cylinder, even though no sensors are integrated into it, can be inferred from the sensors in the hydraulic cylinder surrounding the seal. A classification technique analyzes the effects of the different conditions of a seal on the behavior of the entire system and, thus, the system-level sensor values. The results show that patterns caused by the different conditions can be detected within the data captured with surrounding sensors.

In addition to these contributions, this work also has limitations. We assume that the sensors in the environment record patterns that affect a system component. This can not be proven by the technical experiment presented in this work. In addition, the demarcation of a system in general is not clearly defined. This requires a case-by-case analysis. Furthermore, the application of machine learning also has shortcomings compared to the analysis of physical coherences. The analysis of cause-effect relationships using mathematical models can also be feasible in some cases, however, without the need for collecting training data. However, as with the analysis of physical relationships between components, the sensors used must deliver reliable and stable values over time to ensure the quality of the classification model. A systematic shift in sensor readings over time or a changing sensor configuration can result in decreasing performance.

Nevertheless, we are convinced that this concept provides an exciting research approach. In the next steps, it remains to analyze whether the quality of this approach is comparable to sensor-based condition monitoring in use cases where sensors can be equipped. Thus, our proposed concept could potentially replace sensors within components, which could lead to a decrease in overall system costs.

Furthermore, it has to be investigated which effects the behavior of a system component has on the behavior of other system components. Thus, the concept could be extended if the observation of other (observable) components provides additional insights on the (unobservable) target component. This would be a mixture of the traditional approach (observation of individual components to analyze the overall system conditions) and the concept presented in this work.

In addition, it can be analyzed how the quality of this approach, compared with sensing the component itself, changes for other use cases as well as for other system boundaries.

Our presented use case could also be extended by increasing the granularity of the failure classes leading to more detailed insights.

Finally, we are convinced that our concept can be used in many applications and leads to a broader understanding about system components. This holds the 
potential to make a whole range of applications more transparent and reliable.

\section{References}

[1] F. Civerchia, S. Bocchino, C. Salvadori, E. Rossi, L. Maggiani, and M. Petracca, "Industrial Internet of Things monitoring solution for advanced predictive maintenance applications," Journal of Industrial Information Integration, vol. 7, pp. 4-12, 2017.

[2] J. Gubbi, R. Buyya, S. Marusic, and M. Palaniswami, "Internet of Things (IoT): A vision, architectural elements, and future directions," Future Generation Computer Systems, vol. 29, no. 7, pp. 1645-1660, 2013.

[3] S. A. Macskassy and F. Provost, "A Brief Survey of Machine Learning Methods and their Sensor and IoT Applications," International Conference on Information, Intelligence, Systems \& Applications (IISA), pp. 172-175, 2017.

[4] A. K. Jardine, D. Lin, and D. Banjevic, "A review on machinery diagnostics and prognostics implementing condition-based maintenance," Mechanical Systems and Signal Processing, vol. 20, no. 7, pp. 1483-1510, 2006.

[5] O. Uluyol, G. Parthasarathy, W. Foslien, and K. Kim, "Power Curve Analytic for Wind Turbine Performance Monitoring and Prognostics," Annual Conference of the Prognostics and Health Management Society, no. August, pp. 1-8, 2011.

[6] V. J. Hodge, S. O. Keefe, M. Weeks, and A. Moulds, "Wireless Sensor Networks for Condition Monitoring in the Railway Industry: A Survey," IEEE Transactions on Intelligent Transportation Systems, vol. 16, no. 3 , pp. 1088-1106, 2015.

[7] B. Peeters, J. Maeck, and G. D. Roeck, "Vibration-based damage detection in civil engineering: Excitation sources and temperature effects," Smart Materials and Structures, vol. June, no. October 2014, 2001.

[8] P. Kassanos, S. Anastasova, and G.-Z. Yang, "Electrical and Physical Sensors for Biomedical Implants," in Implantable Sensors and Systems: From Theory to Practice (G.-Z. Yang, ed.), pp. 99-195, Cham: Springer International Publishing, 2018.

[9] P. Kassanos, H. Ip, and G.-Z. Yang, "Ultra-Low Power Application-Specific Integrated Circuits for Sensing," in Implantable Sensors and Systems: From Theory to Practice (G.-Z. Yang, ed.), pp. 281-437, Cham: Springer International Publishing, 2018.

[10] A. J. Thompson and G.-Z. Yang, "Tethered and Implantable Optical Sensors," in Implantable Sensors and Systems: From Theory to Practice (G.-Z. Yang, ed.), pp. 439-505, Cham: Springer International Publishing, 2018.

[11] L. v. Bertalanffy, "An Outline of General System Theory," The British Journal For Philisophy Of Science, vol. 1, no. 2, pp. 134-165, 1950.

[12] P. Checkland, "Systems thinking," in Rethinking Management Information Systems, pp. 45-56, Oxford University Press, 1999.

[13] B. Oshry, Seeing Systems: Unlocking the Mysteries of Organizational Life. Berrett-Koehler Publishers, Inc., 2007.

[14] S. Y. Auyang, "Foundations of Complex-system Theories," in Economics, Evolutionary Biology, and Statistical Physics, pp. 1-404, 1999.
[15] F. C. Fang and A. Casadevall, "Reductionistic and holistic science," Infection and Immunity, vol. 79, no. 4, pp. 1401-1404, 2011.

[16] M. C. Jackson, Systems Thinking: Creative Holism for Managers. John Wiley \& Sons Ltd., 2007.

[17] K. E. Boulding, "General Systems Theory - The Skeleton of Sciene," Management Science, vol. 2, no. 3, pp. 197-208, 1956.

[18] R. L. Ackoff, Creating the Corporate Future: Plan or be Planned For. New York: Wiley, 1981.

[19] Z. K. Peng and F. L. Chu, "Application of the wavelet transform in machine condition monitoring and fault diagnostics: A review with bibliography," Mechanical Systems and Signal Processing, vol. 18, no. 2, pp. 199-221, 2004.

[20] E. P. Carden and P. Fanning, "Vibration Based Condition Monitoring: A Review," Structural Health Monitoring: An International Journal, vol. 3, no. 4, pp. 355-377, 2004.

[21] W. Zhou, T. G. Habetler, and R. G. Harley, "Bearing Condition Monitoring Methods for Electric Machines: A General Review," 2007 IEEE International Symposium on Diagnostics for Electric Machines, Power Electronics and Drives, pp. 3-6, 2007.

[22] S. Bagavathiappan, B. B. Lahiri, T. Saravanan, J. Philip, and T. Jayakumar, "Infrared thermography for condition monitoring - A review," Infrared Physics and Technology, vol. 60, pp. 35-55, 2013.

[23] D. A. Dornfeld and M. DeVries, "Neural Network Sensor Fusion for Tool Condition Monitoring," CIRP Annals - Manufacturing Technology, vol. 39, no. 1, pp. 101-105, 1990.

[24] M. S. H. Bhuiyan, I. A. Choudhury, M. Dahari, Y. Nukman, and S. Z. Dawal, "Application of acoustic emission sensor to investigate the frequency of tool wear and plastic deformation in tool condition monitoring," Measurement: Journal of the International Measurement Confederation, vol. 92, pp. 208-217, 2016.

[25] F. C. Trutt, J. Sottile, and J. L. Kohler, "Online condition monitoring of induction motors," IEEE Transactions on Industry Applications, vol. 38, no. 6, pp. 1627-1632, 2002.

[26] S. Nandi, H. A. Toliyat, and X. Li, "Condition monitoring and fault diagnosis of electrical motors A review," IEEE Transactions on Energy Conversion, vol. 20, no. 4, pp. 719-729, 2005.

[27] I. Alsyouf, "The role of maintenance in improving companies' productivity and profitability," International Journal of Production Economics, vol. 105, no. 1, pp. 70-78, 2007.

[28] K. Peffers, M. Rothenberger, T. Tuunanen, and R. Vaezi, "Design Science Research Evaluation," Design Science Research in Information Systems. Advances in Theory and Practice, pp. 398-410, 2012.

[29] S. Gregor and A. R. Hevner, "Positioning and Presenting Design Science Research for Maximum Impact," MIS Quarterly, vol. 37, pp. 337-355, 122013.

[30] A. R. Hevner, S. T. March, J. Park, and S. Ram, "Design Science in Information Systems Research," MIS Quarterly, vol. 28, no. 1, pp. 75-105, 2004.

[31] S. T. March and G. F. Smith, "Design and natural science research on information technology," Decision Support Systems, vol. 15, no. 4, pp. 251-266, 1995. 
[32] G. C. Cawley and N. L. C. Talbot, "On Over-fitting in Model Selection and Subsequent Selection Bias in Performance Evaluation," Journal of Machine Learning Research, vol. 11, p. 20792107, 2010.

[33] D. Powers, "Evaluation: From Precision, Recall and F-Measure To Roc, Informedness, Markedness \& Correlation," Journal of Machine Learning Technologies, vol. 2, no. 1, pp. 37-63, 2011.

[34] T. S. Baines, H. W. Lightfoot, S. Evans, A. Neely, R. Greenough, J. Peppard, R. Roy, E. Shehab, A. Braganza, A. Tiwari, J. R. Alcock, J. P. Angus, M. Basti, A. Cousens, P. Irving, M. Johnson, J. Kingston, H. Lockett, V. Martinez, P. Michele, D. Tranfield, I. M. Walton, and H. Wilson, "State-of-the-art in product-service systems," Proceedings of the Institution of Mechanical Engineers, Part B: Journal of Engineering Manufacture, vol. 221, no. 10, pp. 1543-1552, 2007.

[35] A. Rytter, Vibrational Based Inspection of Civil Engineering Structures. PhD thesis, Aalborg University, Denmark, 1993. 